\title{
Neuste Zahlen und Übersicht zur Ärztestatistik 2013
}

Die FMH setzt sich seit Jahren für verlässliche und fundierte Statistiken ein. Aus diesem Grund publiziert die Abteilung Daten, Demographie und Qualität DDQ jährlich die neusten Zahlen und Entwicklungen rund um das Thema Ärztestatistik. Detaillierte Informationen zur FMH-Ärztestatistik und zur Mitgliederstatistik sowie zu den wichtigsten Indikatoren finden Sie auf der Website der FMH (www.fmh.ch $\rightarrow$ Services $\rightarrow$ Statistik $\rightarrow$ Ärztestatistik).

Stefanie Hostettler ${ }^{a}$, Esther Kraft ${ }^{b}$

a Dr. sc. ETH Zürich, Daten, Demographie und Qualität DDQ FMH

b lic. rer. oec., Leiterin Daten, Demographie und Qualität DDQ FMH

* Zur besseren Lesbarkeit wird in der Regel die männliche Form verwendet; Frauen sind mitgemeint.

Korrespondenz:

FMH/Abteilung DDQ

Elfenstrasse 18

CH-3000 Bern 15

Tel. 0313591111

ddq[at]fmh.ch

\section{Einleitung}

Die Rahmenbedingungen und Anforderungen des Schweizer Gesundheitswesens befinden sich in einem kontinuierlichen Wandel. Damit das Gesundheitssystem auch künftig auf einem qualitativ hochstehenden Niveau funktioniert, sind Entwicklungen und Tendenzen aufzuzeigen und entsprechende Massnahmen und Anpassungen zu ergreifen. In dieser Hinsicht bildet die FMH-Ärztestatistik eine relevante Datenquelle, um Grundlagen und Indikatoren in der Demographie- und Versorgungsforschung zu erarbeiten. Nicht nur für die Forschung, sondern auch für eine evidenzbasierte Gesundheitspolitik sind die Daten der FMH-Ärztestatistik zentral.

Die FMH-Ärztestatistik wird zu jedem Jahresende (Stichtag 31. Dezember) neu erstellt und umschreibt unter anderem die wichtigsten ärztedemographischen Merkmale wie das Alter, das Geschlecht, die Nationalität und den Ort der Berufsausübung. Als

\section{Abbildung 1}

Übersicht der Anzahl berufstätiger Ärzte von 1960 bis 2013.

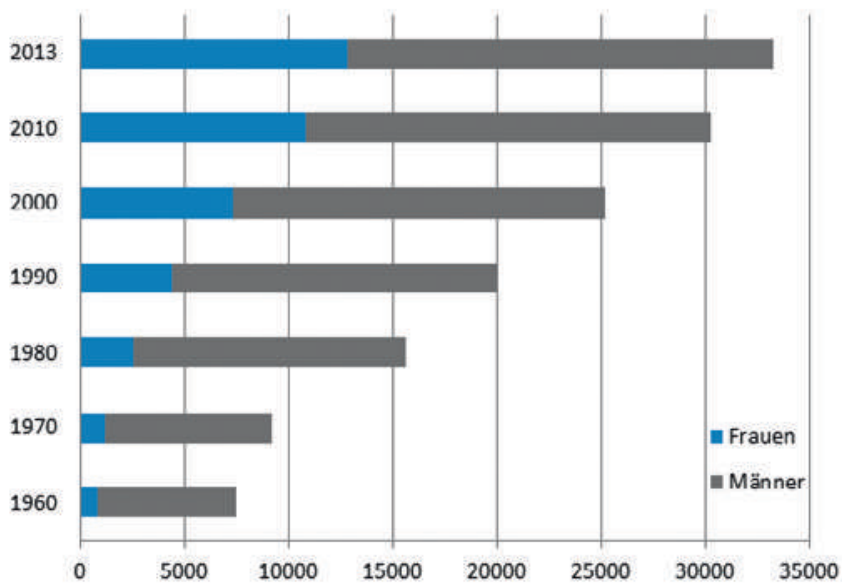

Grundlage dient die Datenbank der FMH mit soziodemographischen Angaben von über 33000 Ärzten*. Die Analysen aus dieser vollzähligen Ärztedatenbank befinden sich im ersten Teil des Artikels. In einem zweiten Teil sind Angaben zur Berufstätigkeit aufgeführt, welche mittels Umfrage auf dem Mitgliederportal myFMH von mehr als 10000 Ärzten selbst deklariert wurden.

Der vorliegende Artikel beinhaltet lediglich eine Auswahl von Inhalten. Weiterführende Informationen in Form von Tabellen, Grafiken, Flyern sowie Ärztestatistiken vergangener Jahre und Indikatoren sind auf der FMH-Website (www.fmh.ch $\rightarrow$ Services $\rightarrow$ Statistik $\rightarrow$ Ärztestatistik) aufgeführt. Ebenfalls dort steht ein Online-Abfragetool zur Verfügung, wo sich mit wenigen Mausklicken die gewünschten Informationen abfragen, als Tabelle oder Grafik darstellen sowie speichern und ausdrucken lassen.

\section{Ärzteschaft im Wachstum}

Im Jahr 2013 waren 33242 Ärzte in der Schweiz berufstätig, was im Vergleich zum Vorjahr einer Zunahme von 4,3 Prozent entspricht. Der Frauenanteil ist in den letzten Jahren kontinuierlich gestiegen und liegt aktuell bei 38,6 Prozent (12816 Frauen, 20426 Männer). Die Zunahme ist bei den Frauen mit 7,1 Prozent höher als bei den Männern (2,7 Prozent), was die Entwicklung des Frauenanteils bei den Studierenden der Humanmedizin der letzten Jahre widerspiegelt. Wie in den Jahren zuvor schlossen wiederum mehr Frauen als Männer das Studium der Humanmedizin ab (541 Abschlüsse im Masterstudium, davon 56,4 Prozent Frauen; 646 Abschlüsse im Doktorat, davon 53,1 Prozent Frauen) [1].

Gerne steht Ihnen die Abteilung DDQ für weiterführende Auswertungen und Fragen zur Verfügung. Kontakt: ddq[at]fmh.ch / 0313591111. 
** Die Angaben zur Ärztedichte gelten für das Jahr 2012, da die Bevölkerungszahlen des Bundesamts für Statistik (BFS) zum Zeitpunkt der Publikation der Ärztestatistik noch nicht verfügbar sind

\section{Mehr als die Hälfte der Ärzte ist im ambulanten Sektor tätig}

52,8 Prozent der Ärzte sind hauptberuflich im ambulanten Sektor tätig, 45,5 Prozent im stationären und 1,7 Prozent in einem anderen Sektor (Versicherungen, Verbände usw.). Der Frauenanteil ist im stationären Bereich (43,3 Prozent) höher als im ambulanten Sektor (34,8 Prozent). Ärzte in der Schweiz arbeiten hauptsächlich in einem Sektor (89,5 Prozent), ein kleiner Teil der Ärzteschaft ist in mehreren Sektoren tätig (10,5 Prozent). Von diesen arbeitet der grösste Teil (84,1 Prozent) hauptberuflich im ambulanten Sektor und führt zusätzlich eine Nebentätigkeit im stationären Bereich aus, zum Beispiel als Belegärzte.

Ein ähnliches Bild präsentiert sich bei der Anzahl Arbeitsorte aus: Die Mehrheit der Ärzteschaft (86,4 Prozent) ist an einem einzigen Arbeitsort tätig.

\section{Abbildung 2}

Ärzte nach Sektoren (Hauptberufstätigkeit) 2013.

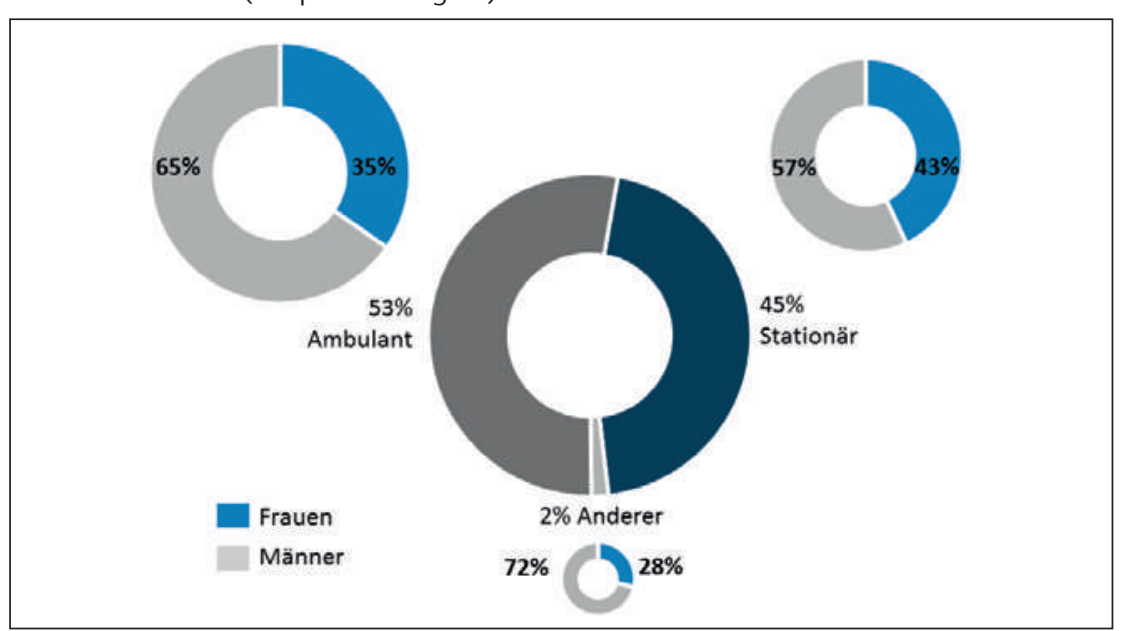

11,1 Prozent arbeiten an zwei Orten und lediglich 2,5 Prozent an drei oder mehr Orten. Davon sind rund dreimal mehr Männer (75 Prozent) als Frauen (25 Prozent), die an mehr als einem Arbeitsort tätig sind.

\section{Ärztedichte nach Kanton}

Durchschnittlich arbeiten 4,0 Ärzte pro 1000 Ein-

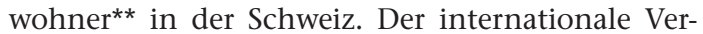
gleich zeigt, dass die Schweiz bezüglich der Ärztedichte über dem Durchschnitt der OECD-Länder liegt (2011: 3,2 Ärzte pro 1000 Einwohner) [2]. Griechenland hatte 2011 mit 6,1 Ärzten pro 1000 Einwohner die höchste Ärztedichte pro Kopf, Indonesien die niedrigste mit 0,2 Ärzten.

Schweizer Kantone mit den höchsten Dichten an Ärzten sind Basel-Stadt (9,2 Ärzte pro 1000 Einwohner), Genf $(6,1)$ und Zürich $(4,8)$. Die tiefsten Ärztedichten weisen Uri (1,6 Ärzte pro 1000 Einwohner), Appenzell I.R. $(1,7)$ und Obwalden $(2,1)$ auf. Im ambulanten Sektor finden sich 0,9 Grundversorger und 1,1 Spezialisten pro 1000 Einwohner. Die Dichte an Grundversorgern ist mit 0,3 deutlich tiefer im stationären Sektor, wo die Dichte 0,9 Spezialisten pro 1000 Einwohner beträgt.

\section{Tabelle 1}

Übersicht der berufstätigen Ärzte nach Sektoren (Hauptberufstätigkeit) 2013.

\begin{tabular}{llll} 
& Frauen & Männer & Total \\
\hline Ambulanter Sektor & 6109 & 11445 & 17554 \\
\hline Stationärer Sektor & 6548 & 8579 & 15127 \\
\hline Anderer Sektor & 159 & 402 & 561 \\
\hline Total & 12816 & 20426 & 33242
\end{tabular}

Abbildung 3

Ärztedichte nach Kanton 2012 (Anzahl Ärzte pro 1000 Einwohner).

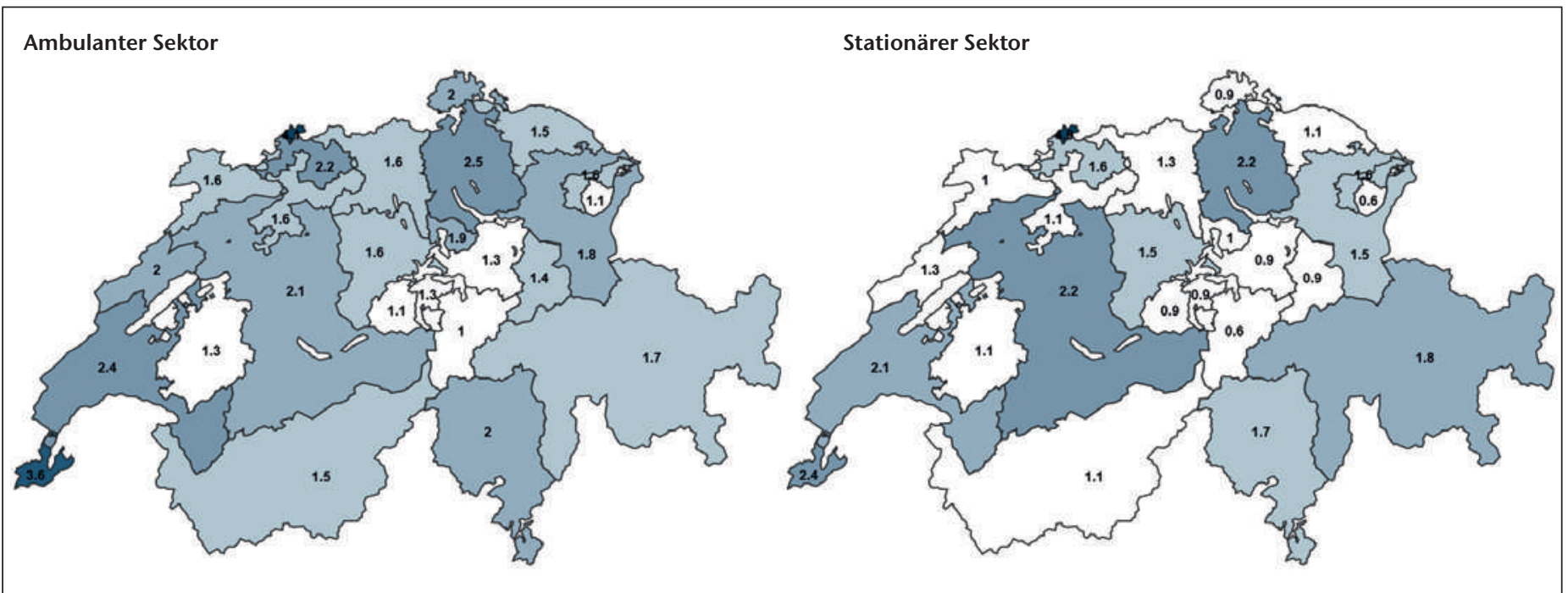




\section{Allgemeine Innere Medizin ist das häufigste medizinische Fachgebiet}

Die Allgemeine Innere Medizin (27,8 Prozent), Psychiatrie und Psychotherapie (12,1 Prozent) und Gynäkologie und Geburtshilfe (5,6 Prozent) sind die häufigsten Fachrichtungen. Im ambulanten Sektor zeigt sich dieselbe Rangfolge: Die grösste Gruppe

Tabelle 2

Übersicht der Ärzte nach Fachrichtung (Hauptberufstätigkeit) 2013.

\begin{tabular}{|c|c|c|c|}
\hline & Frauen & Männer & Total \\
\hline Allergologie/Immunologie & 52 & 99 & 151 \\
\hline Allgemeine Innere Medizin & 2636 & 5244 & 7880 \\
\hline Anästhesiologie & 558 & 797 & 1355 \\
\hline Angiologie & 41 & 131 & 172 \\
\hline Arbeitsmedizin & 37 & 69 & 106 \\
\hline Chirurgie & 177 & 931 & 1108 \\
\hline Dermatologie und Venerologie & 229 & 268 & 497 \\
\hline Endokrinologie/Diabetologie & 70 & 113 & 183 \\
\hline Gastroenterologie & 34 & 292 & 326 \\
\hline Gynäkologie und Geburtshilfe & 880 & 722 & 1602 \\
\hline Hämatologie & 48 & 112 & 160 \\
\hline Handchirurgie & 37 & 133 & 170 \\
\hline Herz- und thorakale Gefässchirurgie & 5 & 78 & 83 \\
\hline Infektiologie & 49 & 103 & 152 \\
\hline Intensivmedizin & 153 & 358 & 511 \\
\hline Kardiologie & 93 & 557 & 650 \\
\hline Kinder- und Jugendmedizin & 866 & 693 & 1559 \\
\hline Kinder- und Jugendpsychiatrie & 377 & 226 & 603 \\
\hline Kinderchirurgie & 24 & 50 & 74 \\
\hline KI. Pharmakologie und Toxikologie & 17 & 25 & 42 \\
\hline Med. Onkologie & 101 & 192 & 293 \\
\hline Medizinische Genetik & 14 & 10 & 24 \\
\hline Mund-, Kiefer- + Gesichtschirurgie & 7 & 80 & 87 \\
\hline Nephrologie & 163 & 367 & 530 \\
\hline Neuropathologie & 3 & 13 & 16 \\
\hline Nuklearmedizin & 12 & 55 & 67 \\
\hline Ophthalmologie & 369 & 566 & 935 \\
\hline ORL & 110 & 343 & 453 \\
\hline Orthopädische Chirurgie & 73 & 874 & 947 \\
\hline Pathologie & 108 & 122 & 230 \\
\hline Pharmazeutische Medizin & 20 & 37 & 57 \\
\hline Phys. Med. u. Rehabilitation & 57 & 128 & 185 \\
\hline Plastische Chirurgie & 43 & 129 & 172 \\
\hline Pneumologie & 48 & 217 & 265 \\
\hline Praktische/r Ärztin/Arzt & 587 & 629 & 1216 \\
\hline Prävention und Gesundheitswesen & 36 & 43 & 79 \\
\hline Psychiatrie und Psychotherapie & 1427 & 1999 & 3426 \\
\hline Radiologie & 204 & 564 & 768 \\
\hline Radio-Onkologie/Strahlentherapie & 40 & 66 & 106 \\
\hline Rechtsmedizin & 18 & 32 & 50 \\
\hline Rheumatologie & 106 & 330 & 436 \\
\hline Tropen- und Reisemedizin & 8 & 22 & 30 \\
\hline Urologie & 27 & 262 & 289 \\
\hline
\end{tabular}

bilden die Grundversorger (Allgemeine Innere Medizin 34,3 Prozent), gefolgt von den Psychiatern (14,4 Prozent) und den Gynäkologen (6,3 Prozent). Im stationären Bereich ist ebenfalls die Allgemeine Innere Medizin mit 17,7 Prozent an der Spitze, an zweiter Stelle hingegen ist die Anästhesiologie (9,7 Prozent), gefolgt von der Psychiatrie und Psychotherapie (8,4 Prozent).

Der Frauenanteil ist in den Fachrichtungen Kinder- und Jugendpsychiatrie $(62,4$ Prozent), Kinderund Jugendmedizin (55,5 Prozent) und Gynäkologie und Geburtshilfe (54,9 Prozent) am höchsten. Die Männer sind im Vergleich zu den Frauen in den chirurgischen Fachgebieten deutlich mehr vertreten (Herz- und thorakale Gefässchirurgie 94,0 Prozent, Orthopädische Chirurgie 92,3 Prozent, Neurochirurgie 86,9 Prozent, Chirurgie 84,0 Prozent).

\section{Altersstruktur der Ärzteschaft}

Das Durchschnittsalter der Ärzte in der Schweiz beträgt 48,8 Jahre (vgl. Tabelle 3). Ärzte im ambulanten Sektor (53,7 Jahre) sind durchschnittlich 10 Jahre älter als ihre Kollegen im stationären Sektor (43,0 Jahre). Dieser Altersunterschied zwischen den Sektoren ist hauptsächlich durch den Anteil und die Weiterbildung der Assistenzärzte bedingt, welcher vorwiegend in Spitälern stattfindet. Die Ärzte sind bei Abschluss der Weiterbildung bzw. bei Erwerb des Facharzttitels durchschnittlich 37 Jahre alt.

Die Abbildung 4 zeigt die geschlechterspezifische Altersverteilung der Ärzteschaft. Unter 35 Jahren sind die Frauen in der Überzahl. Ab dem 40. Lebensjahr sind zurzeit mehr Männer als Frauen berufstätig. Aufgrund der Frauenmehrheit bei den Studierenden und bei der jungen Ärzteschaft ist davon auszugehen, dass sich der wachsende Frauenanteil in den kommenden Jahren entsprechend in der geschlechtsspezifischen Altersstruktur widerspiegeln wird.

\section{Einteilung der Ärzteschaft nach Funktion}

Von den 17554 Ärzten im ambulanten Sektor sind 84,3 Prozent Praxisinhaber oder -teilhaber. 3,5 Prozent sind als Praxisassistent oder Praxisfacharzt angestellt. Den restlichen Ärzten kommt eine andere Funktion zu. Im stationären Sektor bilden die Assistenten in Weiterbildung mit 50 Prozent die grösste Gruppe. Erwartungsgemäss reduziert sich die Anzahl Ärzte mit steigender Hierarchiestufe. So sind im stationären Sektor 20,1 Prozent als Oberarzt,

\section{Tabelle 3}

Durchschnittsalter (in Jahren) der Ärzteschaft 2013.

\begin{tabular}{llll} 
& Frauen & Männer & Alle \\
\hline Ambulanter Sektor & 50,6 & 55,3 & 53,7 \\
\hline Stationärer Sektor & 39,7 & 45,5 & 43,0 \\
\hline Alle & 45,0 & 51,2 & 48,8
\end{tabular}


Abbildung 4

Geschlechtsspezifische Altersstruktur der Ärzte 2013.

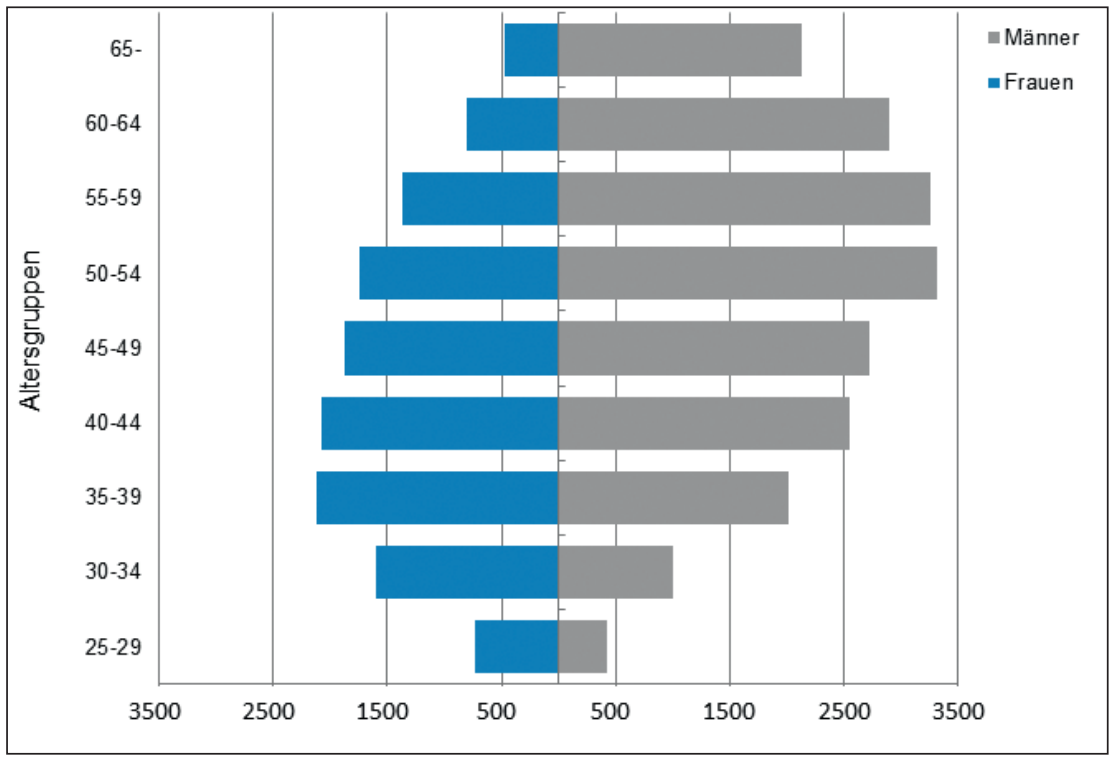

Abbildung 5

Anteil ausländischer Arztdiplome 2013.

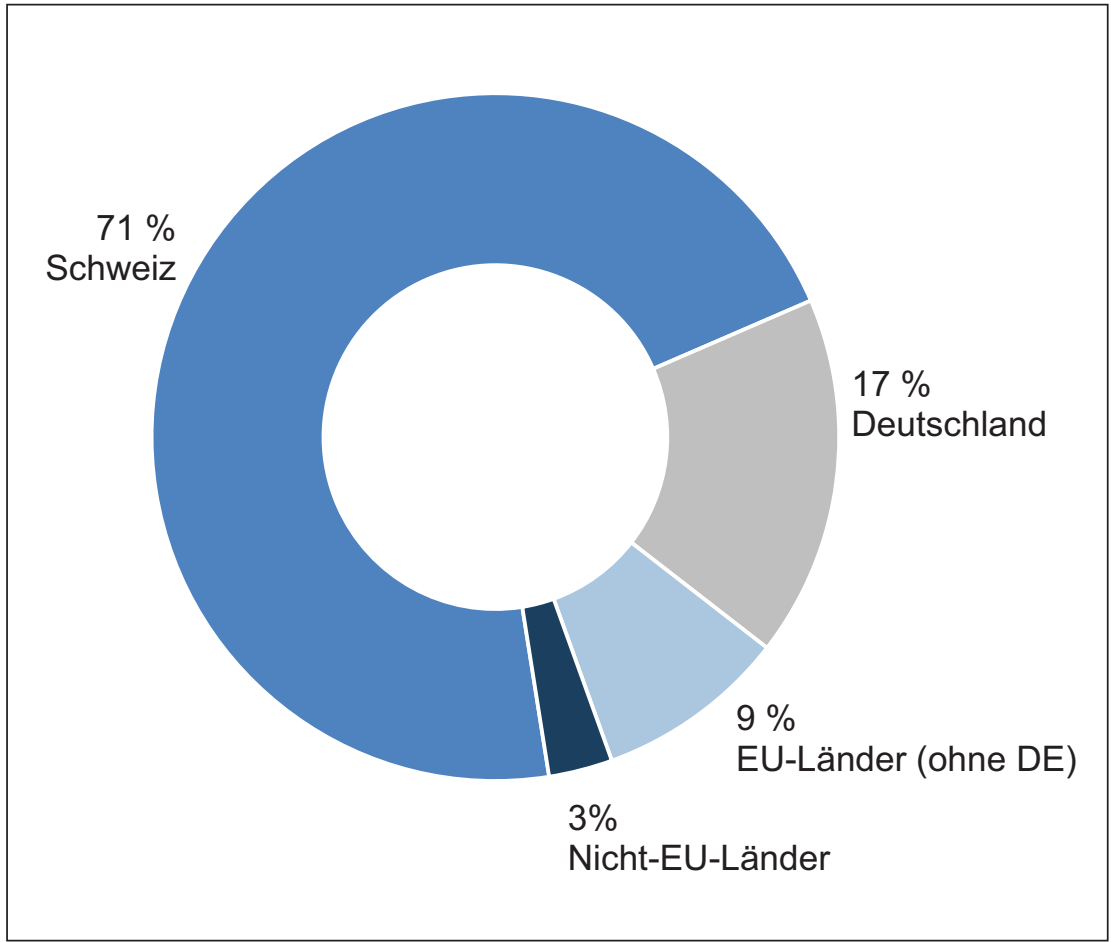

Tabelle 4

Durchschnittliches Arbeitspensum in Halbtagen pro Woche nach Sektor und Geschlecht 2013 (N = 6432).

\begin{tabular}{llll} 
& Frauen & Männer & Total \\
\hline Ambulanter Sektor & 6,8 & 8,8 & 8,2 \\
\hline Stationärer Sektor & 8,8 & 10,1 & 9,5 \\
Anderer Sektor & 7,8 & 9,0 & 8,6 \\
Total & 7,4 & 9,2 & 9,0
\end{tabular}

12,4 Prozent als Leitender Arzt und 10,2 Prozent als Chefarzt tätig. Einzig bei den Assistenzärzten überwiegt der Frauenanteil (56,7 Prozent), danach nimmt er laufend ab: bei den Oberärzten 42,1 Prozent, bei den Leitenden Ärzten 22,4 Prozent und bloss 10,3 Prozent bei den Chefärzten.

\section{Ausländische Arztdiplome}

In der Schweiz sind 9756 Ärzte mit einem ausländischen Studienabschluss in Humanmedizin tätig (Abb. 5). Davon sind 56,3 Prozent im stationären, 41,5 Prozent im ambulanten und 2,2 Prozent in einem anderen Bereich tätig. Die Mehrheit der Fachkräfte aus dem Ausland stammt aus Deutschland (16,9 Prozent) oder aus den übrigen EU-Ländern (9,1 Prozent). Im Vergleich zum Vorjahr ist der Anteil berufstätiger Ärzte mit ausländischem Diplom um 1,8 Prozent auf 29,4 Prozent gestiegen.

Von den insgesamt 1433 Verleihungen für Facharzttitel im Jahr 2013 gingen 37 Prozent an Ärzte mit einem ausländischen Arztdiplom und aus folgenden Herkunftsländern: Deutschland 68,6 Prozent, Österreich 10,2 Prozent, Italien 6,4 Prozent und andere Länder 14,8 Prozent (Quelle: SIWF).

\section{Höheres Arbeitspensum im stationären Bereich}

Die Angaben zum Arbeitspensum und alle Auswertungen im nun folgenden zweiten Teil des Artikels basieren auf Daten, welche die Ärzte mittels Umfrage auf dem Mitgliederportal myFMH selbst deklarieren konnten. Die Datengrundlage wurde hinsichtlich eines Selektionsfehlers für die Faktoren Sektor, Geschlecht und Fachrichtung geprüft und ist mit derjenigen der Gesamtpopulation vergleichbar. Wo die Abweichung zwischen Stichprobe und Gesamtpopulation mehr als 10 Prozent beträgt, wurde eine Gewichtung vorgenommen (Frauen im stationären Sektor).

Die Angaben zum Arbeitspensum wurden in Zusammenarbeit mit NewIndex validiert [3]. Die Analyse ergab, dass die selbstdeklarierten FMHDaten aufgrund der guten Übereinstimmung mit den Daten der Leistungsabrechnung verlässliche und repräsentative Aussagen zulassen.

Ärztinnen und Ärzte arbeiteten 2013 durchschnittlich 9,0 Halbtage (Tabelle 4) pro Woche. Im ambulanten Sektor beträgt das durchschnittliche Arbeitspensum mit 8,2 Halbtagen anderthalb Halbtage weniger als im stationären Bereich (9,5 Halbtage). Das durchschnittliche Arbeitspensum der Ärztinnen (ambulant: 6,8; stationär: 8,8 Halbtage) ist tiefer im Vergleich zu demjenigen ihrer männlichen Kollegen (ambulant: 8,8; stationär: 10,1 Halbtage). In den vergangenen fünf Jahren hat sich dieses Muster nicht wesentlich verändert.

Während im stationären Bereich die Mehrheit der Ärzteschaft (80,0 Prozent) Vollzeit arbeitet, beträgt dieser Anteil im ambulanten Bereich mit 


\section{Online-Abfragetool der FMH-Ärztestatistik}

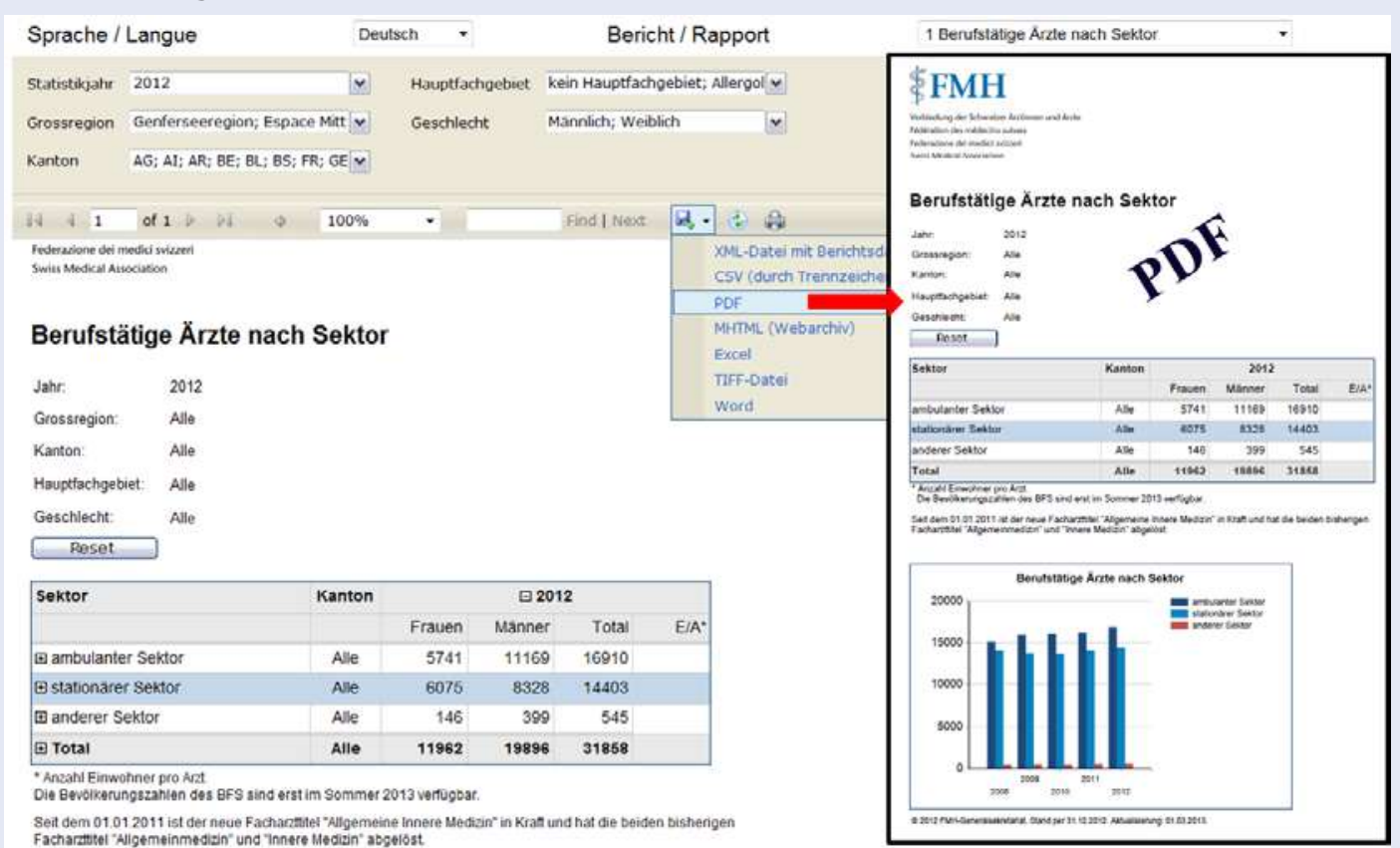

Auf der Website www.fmh.ch unter Services $\rightarrow$ Statistik $\rightarrow$ Ärztestatistik steht das Online-Abfragetool zur Verfügung, um die gewünschten Informationen zur Ärztestatistik selber abzufragen. Die Ergebnisse lassen sich ausdrucken oder als verschiedene Dateiformate (pdf, xIsx, csv usw.) speichern und exportieren. Bei Fragen zur Nutzung des Abfragetools und weiteren Anfragen steht Ihnen die Abteilung Daten, Demographie und Qualität gerne zur Verfügung: ddq[at]fmh.ch, Tel. 0313591111.

56,6 Prozent deutlich weniger. Abbildung 6 zeigt, dass dieser Unterschied zwischen den Sektoren hauptsächlich geschlechterspezifisch bedingt ist. Während im ambulanten Bereich 31,0 Prozent der Männer Teilzeit arbeiten, sind es bei den Frauen mit 73,2 Prozent deutlich mehr. Ärztinnen bevorzugen hauptsächlich ein Arbeitspensum, welches einem Beschäftigungsgrad von rund 60 bis 80 Prozent entspricht. Im stationären Bereich arbeitet die Mehrheit der Ärztinnen (59,7 Prozent) und Ärzte (87,7 Prozent) Vollzeit. Dies liegt womöglich daran, dass die Weiterbildung der Ärzteschaft vor allem im stationären Bereich erfolgt und während dieser Karrierephase das Arbeitspensum seltener reduziert werden kann.

Abbildung 6

Stellenprozente nach Geschlechterverteilung und Sektoren 2013.
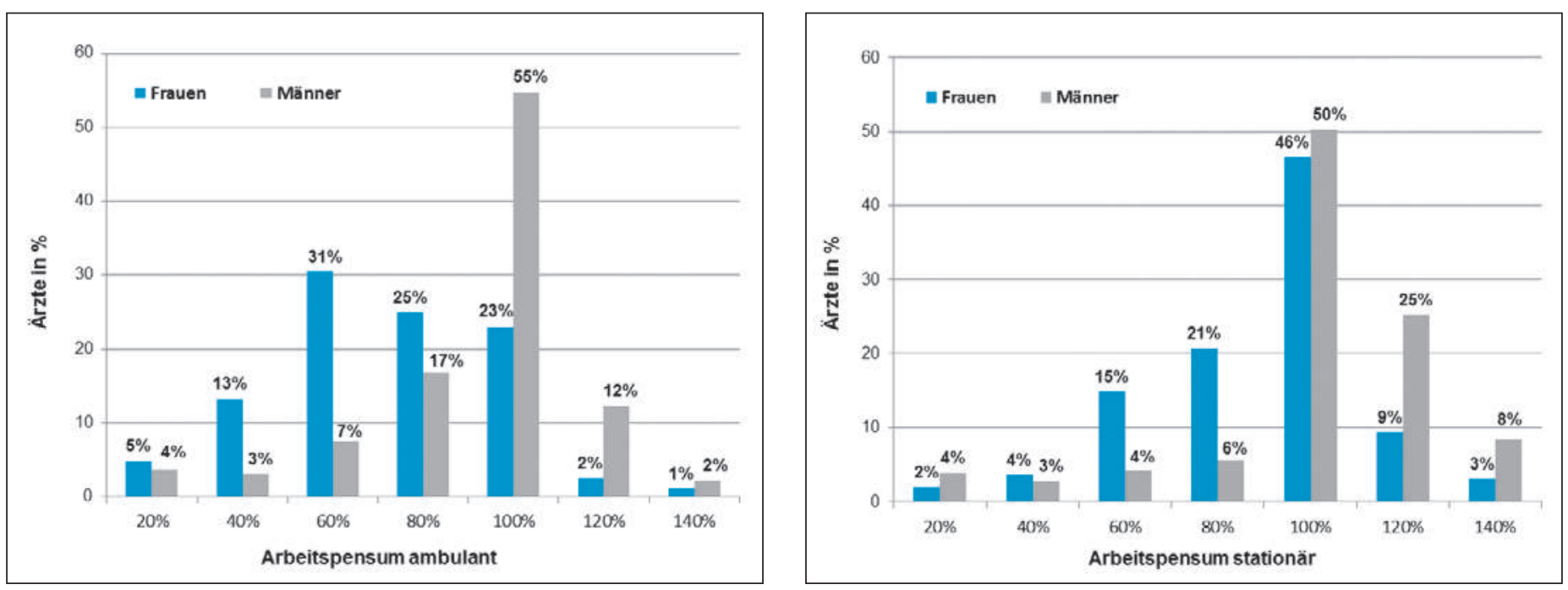


\section{Praxisstruktur und Ärztenetzwerk}

Ärzte im ambulanten Sektor sind vorwiegend in Einzelpraxen tätig (58,6 Prozent). Dieser Anteil hat seit 2008 rund 5 Prozent abgenommen. Gründe für diesen Trend könnten im Wunsch nach mehr Teamarbeit und Teilzeitarbeit sowie nach einem Angestelltenverhältnis liegen.

Im Vergleich zu den Frauen (49,7 Prozent) sind Männer öfter in Einzelpraxen (62,9 Prozent) tätig. Bei den Doppel- und Gruppenpraxen ist der Frauenanteil (50,3 Prozent) im Vergleich zum Männeranteil (37,1 Prozent) höher.

48,5 Prozent der ambulant tätigen Ärzte sind gemäss Selbstdeklaration einem Ärztenetzwerk angeschlossen. Während 50,6 Prozent der männlichen Ärzte in einem Netzwerk vertreten sind, liegt der Anteil bei ihren Kolleginnen mit 43,7 Prozent etwas tiefer. Der Gesamtanteil der in einem Netzwerk tätigen Ärzte blieb im Vergleich zum Vorjahr unverändert.

\section{Schlussbemerkung}

Die dargestellten Ergebnisse der Ärztestatistik 2013 ermöglichen eine differenzierte Übersicht zur Ärzteschaft in der Schweiz und bilden eine evidenzbasierte Grundlage für gesundheitspolitische Entscheidungen. Basierend auf dieser verlässlichen und umfassenden Grundlage strebt die FMH weitere Verbesserungen an (z.B. die Unterteilung des ambulanten Sektors in spitalambulant und praxisambulant). In dieser Hinsicht sollen auch gemäss dem Bericht «Gesundheit 2020» [4] die gesundheitspolitischen Prioritäten für die kommenden Jahre darin liegen, die Transparenz zu erhöhen, lückenhafte Statistiken zu schliessen und valide Daten zur Qualität medizinischer Leistungen zu erfassen. Mit der Ärztestatistik oder der Schweizerischen Akademie für Qualität in der Medizin SAQM verfügt die FMH über geeignete Instrumente zur Erreichung und zur Weiterentwicklung dieser Ziele. Ohne die Mitarbeit der FMH-Mitglieder wäre dies nicht möglich - die FMH bedankt sich an dieser Stelle für Ihr Engagement und hofft, auch weiterhin auf Ihre Unterstützung zählen zu können.

\section{FMH-Ärztestatistik: gute Datenqualität dank Ihnen!}

Für eine gute Datenqualität benötigt die FMH die Unterstützung aller FMH-Mitglieder. Während das Ausfüllen und die Kontrolle des Fragebogens zur Berufstätigkeit nur wenige Minuten beanspruchen, ist die Wirkung für die Ärzteschaft dagegen von grosser Tragweite. Registrieren oder loggen Sie sich auf dem Mitgliederportal myFMH ein und Sie finden den Fragebogen auf der Startseite. Bei Fragen unterstützen wir Sie gerne.

E-Mail: myfmh[at]fmh.ch, Helpline: 0313591259.

Die FMH bedankt sich bei allen, die ihre Daten auf myFMH ausgefüllt oder kontrolliert haben, für die wertvolle Mitarbeit und für die Ermöglichung einer aussagekräftigen Ärztestatistik.

\section{Referenzen}

1 www.bfs.admin.ch/bfs/portal/de/index/themen/15/06/ data/blank/02.html

2 OECD (2013), Health at a Glance 2013: OECD Indicators, OECD Publishing. http://dx.doi. org/10.1787/health_glance-2013-en

3 Hostettler S, Laffranchi R, Kraft E. Ärzteschaft in der Schweiz - Fokus Arbeitspensum. Schweiz Ärztezeitung. 2013;95(50):1891-5.

4 www.bag.admin.ch/gesundheit2020/index. html?lang=de

\section{Interaktiver Artikel}

Wollen Sie diesen Artikel kommentieren? Nutzen Sie dafür die Kommentarfunktion in der OnlineVersion oder sehen Sie nach, was Ihre Kolleginnen und Kollegen bereits geschrieben haben: www.saez.ch/aktuelle-ausgabe/interaktive-beitraege/ 


\section{Glossar (alphabetische Reihenfolge)}

Ambulante Behandlungen im stationären Sektor Alle Behandlungen, die weder als stationär noch als teilstationär angesehen werden.

\section{Ambulanter Sektor}

Zum ambulanten Sektor gehören «Konsultations- und Behandlungstätigkeiten von Ärztinnen und Ärzten in Einzel- oder Gruppenpraxen. Die Patienten werden in der Regel ambulant oder im Haus des Patienten behandelt (Hausbesuche). Inbegriffen sind auch Konsultationstätigkeiten von Privatärzten in Krankenhäusern sowie Tätigkeiten in Kliniken, die Unternehmen, Schulen, Altersheimen, Gewerkschaften und Wohltätigkeitsvereinen angeschlossen sind.» (Quelle: BFS)

Dies gilt zum Beispiel für Ärzte in einer Praxis.

\section{Anderer Sektor}

Zum anderen Sektor gehören Tätigkeiten der Ärztinnen und Ärzte, die weder zum ambulanten noch zum stationären Sektor gehören, zum Beispiel Professor, Dozent, Angestellter bei Versicherungen ...

\section{Arbeitspensum}

Das Arbeitspensum wird in Halbtagen angegeben. Ein Halbtag entspricht einem Arbeitsvolumen von 4 bis 6 Stunden. Eine Vollzeitstelle wird definiert als ein durchschnittliches Wochen-Arbeitspensum von 10 Halbtagen.

Ärzte im ambulanten Sektor

Die Ärztin/der Arzt, die/der seine Hauptberufstätigkeit im ambulanten Sektor hat.

Ärzte im anderen Sektor

Die Ärztin/der Arzt, die/der seine Hauptberufstätigkeit im anderen Sektor hat.

Ärzte im stationären Sektor

Die Ärztin/der Arzt, die/der seine Hauptberufstätigkeit im stationären Sektor hat.

\section{Ärztenetzwerke}

Ärztenetzwerke sind Organisationen, die von Leistungserbringern gebildet werden und der Grundversorgung dienen. Durch verbindliches Zusammenwirken untereinander, mit netzfremden Leistungserbringern und mit den Kostenträgern, erbringen sie auf die Bedürfnisse der Patientinnen und Patienten ausgerichtete Gesundheitsleistungen. Dieses Zusammenwirken beruht auf vereinbarten Behandlungsprozessen, unternehmerischen Organisationsstrukturen und einer gemeinsamen Betreuungskultur.

Doppel- oder Gruppenpraxis

Nutzung von Apparaten, Einrichtungen, Räumlichkeiten oder Personen durch zwei oder mehr Ärzte.

Einzelpraxis

Nutzung von Apparaten, Einrichtungen, Räumlichkeiten oder Personen durch einen einzigen Arzt.

\section{Hauptberufstätigkeit}

Als Hauptberufstätigkeit gilt der Sektor (ambulant, stationär, anderer), an dem die Ärztin / der Arzt mehrheitlich tätig ist.

\section{Hauptfachgebiet}

Das Hauptfachgebiet eines Arztes ist der Facharzttitel, auf dem der Arzt seinen grössten Anteil der medizinischen Tätigkeit ausführt (gemäss Selbstdeklaration und vordefinierten Regeln).

\section{Kanton}

Es gilt derjenige Kanton, in welchem der Arzt seine Hauptberufstätigkeit ausübt. Wenn keine Angaben vorhanden sind, gilt der Kanton der Kontaktadresse.

\section{Stationäre Behandlung im stationären Sektor} Aufenthalt im Spital von mind. 24 Stunden zur Untersuchung, Behandlung und Pflege; Aufenthalt im Spital von weniger als 24 Stunden, bei dem während einer Nacht ein Bett belegt wird, sowie Aufenthalt im Spital bei Überweisung in ein anderes Spital und bei Todesfall.

\section{Stationärer Sektor}

Zum stationären Sektor zählen «Ärztliche Behandlungen, Diagnosen, Pflege, chirurgische Eingriffe, Analysen, Notfalldienst sowie Tätigkeit in der Aus-, Weiter- und Fortbildung usw. in Krankenhäusern. Zum stationären Sektor gehören auch Wohnheime mit einer sozialen Betreuung rund um die Uhr von Kindern, Betagten und Personengruppen, die auf fremde Hilfe angewiesen sind.» (Quelle: BFS) 